\title{
LA EDUCACIÓN EN MATERIA DE COMUNICACIÓN, UNA ASIGNATURA PENDIENTE
}

\author{
Carmen Marta Lazo
}

(Universidad San Jorge de Zaragoza)

cmarta@usj.es

\section{Resumen:}

La Educación en Materia de Comunicación es un tema con la suficiente relevancia y entidad en la sociedad actual como para estar integrado en el currículo escolar dentro de los denominados ejes transversales. La realidad exige que los escolares aprendan en las aulas a mirar con criterio fundamentado los mensajes que les proporcionan las distintas pantallas con las que diariamente se relacionan. Además, son los propios niños quienes reclaman la necesidad de ese aprendizaje desde la escuela, ya que consideran que sus padres no están cualificados para abordar determinadas cuestiones relativas a las formas de interpretar los contenidos televisivos.

Palabras clave: Educación en comunicación, competencia televisiva, tema transversal, currículo escolar, educación primaria.

\begin{abstract}
:
Education in Communication carries enough weight and magnitude in present society as to be integral part and parcel of our schools' syllabi (if only as an across-thecurriculum subject matter). Reality demands that schoolchildren learn how to look at messages they are daily bombarded with from within the scope of a well-formed criterion. Furthermore, it is the children themselves that claim for the need of such learning at school, since they do not deem their parents as appropriately qualified to tackle certain issues concerning the interpretation of TV contents.
\end{abstract}

Key words: Education in communication, TV competence, across-the-curriculum subject matter, school's syllabus, Primary Studies.

\section{LA ESCUELA COMO CONTEXTO DE ADQUISICIÓN DE COMPETENCIA CO- MUNICATIVA}

a televisión constituye uno de los principales agentes de socialización para los menores. A través de la pantalla, los niños acceden a otros mundos, aprenden nuevos conceptos y generan actitudes distintas a su realidad cotidiana. Sin embargo, que el niño esté o no preparado para saber interpretar de forma integral las imágenes a las que se expone depende de múltiples factores, con un eje en común: la adquisición de competencia comunicativa. 
La alfabetización audiovisual sirve al niño para conocer las herramientas y formas de operar en el campo de la imagen. El desarrollo de la capacidad crítica a la hora de saber discriminar, comparar y explicar las secuencias vistas en televisión supone un paso más que el simple y mero conocimiento de la técnica televisiva.

Ante los beneficios que supone el adiestramiento en el campo de la contextualización y re-narración de los significados, cabe considerar de qué modo hay que preparar al niño para ello y a qué instancia o instancias les compete llevar a cabo esta labor. Los padres serían la primera fuente de contraste de lo visto en televisión por encontrarse en el ámbito en el que tiene lugar el visionado. A pesar de ello, la realidad evidencia que, en muchos casos, los adultos no disponen del tiempo o de la preparación suficiente para ejercer esta importante función. En una investigación abordada con 440 escolares de la Comunidad de Madrid para recabar tendencias en este sentido (Marta Lazo, 2005), advertimos que sólo algo más de la mitad de los niños, un $54 \%$ en concreto, suele conversar con sus padres respecto a los contenidos vistos. Los motivos por los que los adultos no suelen dialogar a este respecto con sus hijos se deben a que no se sienten especialmente capacitados para transmitir una buena información y educación televisiva a sus hijos (Marta Lazo, C.; De Ángel García, 1997). A similares conclusiones se llegan en el estudio titulado La televisión y los niños realizado por el CIS (2000): tan sólo el $42 \%$ de los padres afirmaba que preocuparse por los programas de televisión que ven los niños y adolescentes es una labor exclusiva de los padres. El resto sentían la necesidad de compartir esta función con las propias cadenas de televisión (un 30\%) o con la escuela (un 25\%), al manifestar que no se sienten preparados para ejercer esta tarea en solitario.

En este apartado, nos parece interesante recoger el símil que establece Younis (1993: 175) entre una casa y la mente de los niños. Este autor asegura que, si los educadores (padres y maestros) intervienen conjuntamente, los pequeños amueblarán mejor su mente, porque la capacidad de influir de la televisión dependerá de la cantidad de habitaciones y de la calidad de los muebles (experiencias y conocimientos) que tengan los niños, para los que la ayuda de un adulto en su construcción es básica.

Por tanto, la aportación de la escuela servirá de complemento para ampliar las acciones que desarrollen los padres o iniciarlas, en caso de que no se preocupen de enseñar a sus hijos a ver televisión o de que no acostumbren a compartir el tiempo televisivo ni a dialogar respecto a lo que ven en la pantalla. Sin duda, como veremos en los siguientes epígrafes, la experiencia en las aulas puede llegar a ser enormemente enriquecedora tanto para los niños que no cuentan con directrices de sus padres como para aquéllos que lo trabajan en su hogar, debido a que un refuerzo en el colegio siempre resultará un componente añadido a su formación televisiva.

\section{PRINCIPALES ARGUMENTOS POR LOS QUE LOS NIÑOS SIENTEN LA NE- CESIDAD DE QUE EN LA ESCUELA SE LES ENSEÑE A VER LA TELEVISIÓN}

En el apartado cualitativo de la ya mencionada investigación (Marta Lazo, 2005: 193-280) llevada a cabo en la Comunidad de Madrid con 16 grupos de discusión de niños entre 7 y 12 años, les planteamos de qué modo consideraban el posible apoyo de la escuela para aprender a mirar la televisión. A continuación presentamos de manera clasificada la modalidad de demandas que los escolares solicitaban al sis- 
tema educativo, junto con algunos de los ejemplos de los discursos recogidos, identificados por las características o composición de variables de su grupo de pertenencia:

- Como orientación respecto al contenido que pueden ver, en función de su edad:

- Niños 7-9 años, ven más de dos horas la TV y dialogan con los padres respecto a lo que ven: Sí, porque así todo el mundo del colegio saben lo que pueden ver y lo que no. Sí, porque hay niños de $6^{\circ}$ que ven Crónicas Marcianas.

- Niña 7-9 años, ve menos de dos horas la TV y no dialoga con los padres respecto a lo que ve: Si me enseñan a ver lo que tengo que ver sin palabrotas, sí.

- Niño 10-12 años, ve más de dos horas la TV y no dialoga con los padres respecto a lo que ve: para saber qué tenemos que ver, las cosas de nuestra edad y las que son para todos los públicos.

- Niña 10-12 años, ve menos de dos horas la TV y no dialoga con los padres respecto a lo que ve: Pienso que sería bueno, nos enseñaría las cosas que debemos ver, las que no...

- Como recurso didáctico para el aprendizaje de diferentes materias (contenidos conceptuales):

- Niña 7-9 años, ve menos de dos horas la TV y no dialoga con los padres respecto a lo que ve: Ver películas en inglés, sí.

- Niño 10-12 años, ve más de dos horas la TV y dialoga con los padres respecto a lo que ve: Puede ser interesante porque, así, te enseñan a conocer las cosas.

- Niño 10-12 años, ve más de dos horas la TV y no dialoga con los padres respecto a lo que ve: para aprender cosas de la tele, por ejemplo, de las que salen en documentales y programas de preguntas.

- Como herramienta para plantear demandas al medio de comunicación como ciudadanos o como plataforma para aprender la manera de ver la televisión (contenidos procedimentales):

- Niños 7-9 años, ven más de dos horas la TV y dialogan con los padres respecto a lo que ven: Es que tendrían que poner en la tele, en todos los programas para quien son. En un canal, cosas para pequeños y, en otro canal, cosas para mayores... (plantean que en la televisión debe advertirse a qué público está dirigido cada programa o que se separe la oferta en diferentes canales para evitar confusiones). 
- Niño 10-12 años, ve más de dos horas la TV y dialoga con los padres respecto a lo que ve: A mí, me parece que estaría bien porque hay niños que la ven de muy cerca, así les podrían explicar cómo se ve y qué programas.

- Niña 10-12 años, ve menos de dos horas la TV y no dialoga con los padres respecto a lo que ve: $A$ veces, nos pasamos viendo cosas, te adictas, deberían aconsejar cómo verla. A veces, estás viendo tanto rato la televisión y no sabes ni lo que estás viendo.

- Como medio para aprender lo relativo a los contravalores o forma ilustrativa de conocer las consecuencias negativas que pueden tener determinados comportamientos (contenidos actitudinales):

- Niño 7-9 años, ve menos de dos horas la TV y no dialoga con los padres respecto a lo que ve: Deberían de enseñarnos porque, luego, nos viciamos con la televisión y a nuestros padres no les dejamos verla y la televisión nos come el coco y hay veces que podemos maltratar a nuestros padres.

- Niño 10-12 años, ve más de dos horas la TV y dialoga con los padres respecto a lo que ve: Te enseñan a conocer las cosas que te pueden pasar si te marchas a una de estas cosas que son malas para la salud, te enseñan lo que puedes llegar a hacer.

- Niña 10-12 años, ve menos de dos horas la TV y no dialoga con los padres respecto a lo que ve: Sí, no sólo de la televisión, también de las drogas, tabaco,... porque si nos enseñan a hacer las cosas de una manera las haremos así.

Por otro lado, enfatizamos en que el principal motivo por el que los escolares creen interesante integrar la enseñanza de la televisión en el currículo escolar es, precisamente, la limitación que opinan que tienen sus padres a la hora de explicarles lo que no entienden de la televisión y también porque la escuela supone para ellos un referente importante:

- Niña 10-12 años, ve más de dos horas la TV y dialoga con los padres respecto a lo que ve: Sí, porque hay cosas que no las entiendes y tus padres no saben cómo explicarlas.

- Niña 7-9 años, ve más de dos horas la TV y no dialoga con los padres respecto a lo que ve: Sí, así, nos educan más y sabemos lo que tenemos que hacer.

\section{LA EDUCACIÓN EN MATERIA DE COMUNICACIÓN, OLVIDADA ENTRE LOS TEMAS TRANSVERSALES ACTUALES DE LA EDUCACIÓN PRIMARIA}

Como hemos podido observar, los parámetros por los que los niños estiman oportuno e importante que sea la escuela una fuente de conocimiento para aprender a utilizar los mensajes mediáticos son tan diversos que completan todas las tipologías de contenidos que se asumen desde el sistema educativo actual. Unos aluden al 
plano conceptual, como recursos para la enseñanza de otras materias; otros a la categoría de procedimientos, como aprendizaje de las formas de operar ante el medio televisivo y los programas que desde él se ofrecen; y, en otros casos, se hace referencia a aspectos actitudinales relacionados con comportamientos óptimos o negativos, dependiendo de la forma en que se actúe ante la pantalla y las consecuencias que se desprendan del uso que se haga.

De esta forma, todas las vertientes que exponen los niños conforman la esencia de un aprendizaje integral en materia de comunicación. Así, consideramos que éste debería introducirse como un eje transversal dentro del currículo escolar, con la misma entidad que tienen los temas transversales que actualmente están incorporados en el sistema educativo y que son valorados desde el Ministerio de Educación y Ciencia como cuestiones que reclaman en la época actual una atención primaria. En el mismo documento que se hace constar ese carácter prioritario de los temas transversales, se señalan como ejemplos los siguientes aspectos que estarían contenidos en ellos: los grandes conflictos contemporáneos del mundo como la violencia, las desigualdades, la escasez de valores éticos, el despilfarro, la degradación del medio ambiente o hábitos que atentan contra la salud y se indica que no pueden pasar desapercibidos para el sistema educativo (MEC, 1993).

Los temas en los que se materializan esos contenidos que destaca el MEC y que hoy siguen estando vigentes en el currículo son: Educación moral y cívica; Educación para la paz; Educación para la igualdad de oportunidades entre sexos; Educación para la salud/Educación sexual; Educación medioambiental; Educación del Consumidor; y Educación vial. Sin embargo, en ese listado no se contempla la Educación en materia de comunicación, a pesar de ser un tema relevante en la sociedad actual, debido al grado de influencia que tienen los medios, en especial la televisión, en la vida de los menores. Con este mismo criterio, Aguaded (1999: 112) expone que:

\begin{abstract}
Si la escuela ha de tender a la educación armónica de los alumnos para que adquieran, tanto plena conciencia de sí mismos, como autonomía en su actuación, ¿cómo ignorar la poderosa influencia del medio televisivo y no ayudarles a conocer, comprender, interpretar y usar la televisión? Es necesario por ello que, desde el aula, se aprovechen las indudables potencialidades educativas del medio y que, al mismo tiempo, se favorezca que los chicos y chicas puedan defenderse del acoso manipulativo que con mucha frecuencia -y no siempre de forma patente- está explícito en los mensajes audiovisuales.
\end{abstract}

A este respecto, Pérez Tornero (1994: 44) sostiene que, desde el sistema televisivo, se ha concebido el poder de decisión del usuario como algo prescindible, en pro de una voluntad de manipulación y de los beneficios económicos, entre otras finalidades menos éticas. No obstante, para este autor, el camino está trazado: el usuario sólo tiene que comprender esa situación, habiendo adquirido la competencia televisiva correspondiente, para cambiarla y conseguir una actividad más libre y útil ante la televisión. Además, señala que esta actividad debe proyectarse desde diferentes dimensiones:

1. Desde el punto de vista del espectador: en la selección, el uso y la utilización de los programas. 
La educación en materia de comunicación, una asignatura pendiente

2. Desde el punto de vista del decodificador de los mensajes: teniendo la posibilidad de realizar una lectura crítica, no mistificada y libre de manipulaciones abusivas.

3. Desde el punto de vista del usuario de la televisión: utilizándola como instrumento práctico bien para el aprendizaje, para la indagación o para la comunicación.

4. Desde el punto de vista de la expresión: pudiendo ser el telespectador activo en la confección del mensaje televisivo, sea encontrando el modo de trasladar a él las demandas que se efectúen, o bien participando, de modo directo o indirecto en su elaboración.

A juicio de Pérez Tornero (1994: 138), llegar a la primacía del usuario, obliga a formar en la libertad en el uso del medio: en la libertad que provoca la comprensión inteligente; libertad asociada a la posibilidad de recreación de sentidos y de reinterpretación de los mensajes; libertad, finalmente, para usar-tanto en el terreno privado como público- las potencialidades del medio en función de nuestros proyectos e intereses. Y para ello se requiere una preparación a la que la escuela debe hacer frente.

La televisión es una fuente potencial de adquisición de determinados valores y esta guía tiene tanta trascendencia en los comportamientos de los niños y jóvenes que debe reclamarse al sistema educativo la reconsideración de convertirse en una de sus prioridades como eje transversal. Además, cabe añadir que no todos los valores tienen una dimensión pro-social, sino que en su mayoría pertenecen al polo opuesto de contravalores. Así lo exponen Alonso, Matilla y Vázquez (1995: 135-136), para quienes estos últimos son más habituales en la pantalla que los valores considerados correctos:

Las actitudes y valores que predominan en los programas infantiles, así como en el particular universo de los anuncios publicitarios, se identifican con la competitividad y el individualismo, el recurso a la violencia como medio para resolver conflictos, la estimulación del consumo y de la adquisición de cosas como forma de satisfacer los deseos, etc.

Al contrario de este modelo que, según ellos, refrenda estereotipos permanentes, estos autores defienden la construcción de una cultura infantil que fomente las potencialidades del desarrollo autónomo de los niños y niñas, dentro de un sistema de valores congruentes con el ideal de una sociedad democrática, igualitaria, solidaria, emancipadora, superadora de las desigualdades entre sexos y las razas, y respetuosa con los derechos de mercancías y en esta cultura consideran que la televisión también jugará un papel destacado.

En cuanto a la política educativa que se ha llevado a cabo en los últimos años, García Matilla (2003: 181-182), sostiene que:

No sólo no se ha apoyado, sino que se ha tendido a torpedear el avance de una educación en materia de comunicación... se ha quitado peso a asignaturas vinculadas con una visión amplia de la E.P.C. (Educación para la Comunicación) y se ha dado un peso casi definitivo a la enseñanza de las Tecnologías de la Información y de la Comunicación (TIC), entendiendo éstas como la transmisión de un conoci- 
miento pragmático en el uso de informática, de Internet y de determinadas aplicaciones multimedia. Paralelamente, se ha desactivado en gran medida la orientación crítica para dar paso a un tipo de formación muy complaciente y que en algunos casos ha mostrado casi una fe ciega en el poder de la tecnología para resolver los problemas.

Ante esta situación, García Matilla valora la contrarreforma de los sistemas educativos, ocurrida en el final de los años noventa y principio de la década de los dos mil, como muy negativa en el ámbito de la Educación para los medios, respecto a los avances que se habían alcanzado en décadas anteriores. Como consecuencia, concluye que hoy se hace más necesaria que nunca esa reorientación de la enseñanza como proceso con el que los alumnos han de ser protagonistas de su propio aprendizaje, y por ello es preciso no renunciar a plantear estrategias para una mediación eficaz.

En la actualidad, en el currículo escolar de Primaria, la Educación en materia de comunicación queda restringida a la aportación en contenidos puntuales en algunas áreas curriculares, los cuales están regulados en el Real Decreto 1006/1991, de 14 de junio por el que se establecen las enseñanzas mínimas correspondientes a la Educación Primaria.

\section{LA REALIDAD ACTUAL FUNDAMENTA LA INTEGRACIÓN DEL APRENDI- ZAJE EN MATERIA DE COMUNICACIÓN DENTRO DEL CURRÍCULO ESCOLAR}

Ante los cambios mediáticos, el incremento del caudal informativo y las múltiples posibilidades que permiten los nuevos medios se hace en mayor medida necesaria la formación del alumnado en materia de comunicación. Como apunta Cebrián Herreros (1995: 512), enseñar a ver y a analizar televisión se hace más imprescindible a partir de las tendencias narrativas audiovisuales que incorporan nuevas dificultades a la lectura por la aceleración de la cadencia de planos, por la complejidad del montaje, por el ritmo expositivo y por la densidad de información que se acumula en cada situación.

El desarrollo de la convergencia multimedia ha creado nuevos sistemas expresivos y fórmulas de comunicación, sirviendo de soporte medios tradicionales como la pantalla de televisión, pero con mayor número de posibilidades, tanto por el incremento geométrico de las ofertas como por las nuevas opciones que presentan, por ejemplo las ofertas de las plataformas digitales y por cable. Esta nueva concepción de destinatario, que a su vez es cliente de múltiples servicios a los que puede acceder directamente, genera nuevas necesidades de formación para el ejercicio autónomo y crítico y ese aprendizaje debe plantearse y potenciarse desde la escuela.

De este modo, se trata de formar a los niños para que sepan reconducir la información, reflexionar sobre ella y reconstruir su significado. Al igual que en el colegio se les enseña educación vial y no se pretende que sean guardas de tráfico o aprenden educación para el medio ambiente y la misión no es convertirlos en agentes forestales, también es conveniente educarles en materia de comunicación, no como profesionales pero sí como usuarios avanzados de los medios con oportunidades de expresarse y, por consiguiente, como ciudadanos que tengan posibilidades de acceso y demanda a unas cadenas de televisión y por extensión al resto de medios cuyo fin, al menos en la consideración normativa, es hacer frente al servicio público. 
Con esta finalidad, expondremos a continuación, de forma breve, la propuesta de integración de un tema transversal en el currículo de la enseñanza formal de Primaria, consistente en Educación en materia de comunicación, con el peso que la realidad social exige (Marta Lazo, 2005: 281-314).

\section{PROPUESTA DE UN TEMA TRANSVERSAL DE EDUCACIÓN EN MATERIA DE COMUNICACIÓN}

El motivo por el que nos decantamos por la nomenclatura Educación en materia de comunicación se debe a que se corresponde a la denominación europea, que ha tenido varias designaciones: Educación para los medios, Educomunicación, Educación para la Comunicación, Educación por la Comunicación, Educación para la recepción (crítica, activa), Pedagogía de la Imagen. No obstante, a pesar de las diferentes formas de nombrar esta enseñanza, su significado es similar, en todos los casos (UNESCO, 1984: 8).

Esta propuesta parte de la manifiesta necesidad de establecer en el sistema educativo procesos de enseñanza-aprendizaje donde el principal eje sea la comunicación en todas sus dimensiones. El principal objetivo consiste en establecer una acción integral basada en potenciar la autoridad y la autonomía del alumno en relación con los medios, al tiempo que fomentar la capacidad crítica y creativa del niño, en su interacción con los mensajes mediáticos.

El uso más común que, tradicionalmente, se les ha otorgado a los medios en las aulas ha sido el de soportes complementarios a la enseñanza, desde la histórica pizarra, pasando por el retroproyector y por el vídeo hasta llegar a los multimedia actuales. Esta educación con los medios o su aprovechamiento didáctico para la educación formal supone un primer acercamiento o punto de partida, en la triple dimensión cómo recursos didácticos, cómo objeto de estudio o cómo agentes educativos (Gutiérrez Martín, 1997: 95-98) o, lo que es lo mismo, en las siguientes vertientes de explotación didáctica de los medios: aprender con el medio; conocer e interpretar el medio; y comunicarnos con el medio (Aguaded, 1999: 150-156). Nuestra propuesta iría encaminada hacia una conjunción en el aprendizaje integral de esas tres perspectivas, las cuales hemos concretado en la formación del niño a lo largo de tres estadios: programador, árbitro y creador.

El primer paso consiste en que el niño se convierta en programador de lo que los medios le ofrecen o proponen. Esto lo conseguirá mediante la elección, con criterio autónomo y crítico, de los canales que más le interesen de toda la variedad de medios, soportes y aplicaciones existentes, teniendo como principal referencia el contenido concreto que desea utilizar. Por tanto, no se dispondrá a ver cualquier cosa que se emita en televisión, que esté accesible en la red o en su colección de CDRoms, sino que previamente programará, planificará y dosificará lo que desea mirar o utilizar, conociendo y sabiendo de antemano lo que quiere.

Como segundo escalafón, el niño actuaría como árbitro de lo que el emisor le propone. Tras haber adquirido la competencia comunicativa requerida, ya sabrá leer, con juicio crítico, el significado del mensaje en todas sus dimensiones. Esta experiencia formará parte de su poso de conocimientos mediáticos y le servirá para comparar, en futuras ocasiones, su relación con esa fuente o con otras. Asimismo, si el 
medio permite una interactividad real (o si no la posibilita, intentándolo por otras vías), el niño podrá denunciar aquello que estime inoportuno y proponer otras fórmulas de construcción o alternativas en los contenidos.

Como tercera finalidad, el niño llegará a convertirse en creador, siendo emisor en sentido puro, a través de la producción de espacios de televisión, el diseño de documentos multimedia o la creación de páginas web, entre otros. En este estrato, será cuando pueda aplicar todo lo aprendido, pues será el artífice de producciones en las que tendrá que autocuestionarse si son acordes con las premisas éticas y valores que ha ido adquiriendo en su proceso formativo.

En resumen, la Educación en materia de comunicación que proponemos se iniciaría con el conocimiento de las funciones que cada agente puede operar en el proceso comunicativo para que el alumno sepa cómo poder participar en él y de qué manera protagonizarlo. En primer lugar, mediante la selección autónoma de los soportes y mensajes que le interesan, por lo que tendrá que conocer las posibilidades que el medio de comunicación o aplicación tecnológica le ofrecen y las que no. Para aprender cuáles son sus herramientas y sus funciones y saber cuáles son cada una de las partes del proceso de producción de los mensajes, a través del aprendizaje de la lectura mediática, del significante y el significado, con una mirada o uso crítico de los mismos. Además, el mejor conocimiento de todas las herramientas, lenguajes y códigos se producirá cuando el niño aprenda a manejarlos de manera activa, de esta forma tendrá posibilidades de crear sus propios mensajes en distintos soportes, con el fin de que este conocimiento fruto de su experiencia práctica le sirva para evaluar a posteriori cada una de las acciones y relaciones que mantenga con los medios. De este modo, esta materia sobrepasará las puertas de la escuela, será continua y formará parte de una educación para la ciudadanía, porque seguirá a lo largo de toda la vida del educando.

\section{CONCLUSIONES}

La televisión es un importante agente de socialización en la vida de los menores que hay que considerar y para la que hay que formarles. Los padres estiman que no cuentan con la suficiente preparación como para enseñar a sus hijos de qué manera hay que mirar los mensajes televisivos. Los niños también coinciden en que sus padres no están cualificados para desempeñar esta labor y argumentan que debe ser la escuela la instancia que les enseñe a ver televisión.

Los escolares consideran distintas modalidades de contenidos que pueden enseñarse desde las aulas en relación con el medio televisivo. Las sugerencias de aprendizaje que advierten completan las formas de contenidos instaurados en el sistema educativo, unas se entablan en el eje de los conceptuales, otras en el de los procedimentales y otras en el de los actitudinales. Sus propuestas van desde servir como recurso didáctico para el aprendizaje de distintas disciplinas o utilizarse, precisamente, como medio para aprender a operar ante la televisión, hasta llegar a convertirse en una guía de formación en valores y comportamientos prosociales.

El sistema educativo actual considera los temas transversales como cuestiones de especial relevancia en la época actual. Sin embargo, no contempla entre ellos la Educación en materia de comunicación. La integración de esta materia en el currí- 
culo escolar es fundamental en una sociedad en la que los medios de comunicación son una fuente caudalosa de contravalores. Por ello, hay que enseñar a los niños a reinterpretar el contenido de los mensajes y convertirlos en sujetos autónomos y críticos en su interacción con las pantallas.

Los nuevos medios posibilitan usos distintos a los tradicionales, en los que el menor puede convertirse en cliente de servicios, para los que tiene que estar todavía más preparado, con objeto de saber actuar con un criterio responsable. La Educación en materia de comunicación más completa iría encaminada hacia un aprendizaje integral de todas las funciones que el niño puede desarrollar delante de los medios. Éstas podrían resumirse en las siguientes: conocimiento de las funciones que cada agente en el proceso comunicativo puede operar, aprendizaje de la lectura mediática para una mirada o uso crítico de los contenidos y conocimiento para un uso creativo de las herramientas, lenguajes y códigos, junto con los cambios actitudinales en el comportamiento del niño en su relación con los medios y nuevas tecnologías.

\section{REFERENCIAS BIBLIOGRÁFICAS}

AGUADED, J. I.; DÍAZ GÓMEZ, R. "La formación de telespectadores críticos en educación secundaria”. Revista Latina de Comunicación Social [en línea], n 63 (2008), pp. 121-139. [Consulta: 3 de mayo de 2008]. Disponible en http://www.ull.es/publicaciones/latina/ 2008/12 19 Huelva/Aguaded.html

AGUADED, J. I.; CABERO, J. y SALINAS, J. (Eds.) Diseño, producción y evaluación de medios para la formación. Madrid: Alianza, 2003.

AGUADED, J. I. (2005). "Enseñar a ver la televisión: una apuesta necesaria y posible". Comunicar. Revista Científica Iberoamericana de Comunicación y Educación, (Noviembre 2005), n² 25, pp. 51-55.

- (2003). La educación en medios de comunicación en la transversalidad curricular. En APARICI, R. (coord.). Comunicación Educativa en la Sociedad de la Información. Madrid: UNED.

- (1999). Convivir con la televisión. Familia, educación y recepción televisiva. Barcelona: Paidós.

- (1998) Descubriendo la “caja mágica”. Enseñamos a ver la tele. Huelva: Grupo Comunicar.

ALONSO, M.; MATILLA, L.; VÁZQUEZ, M. Teleniños Públicos, Teleniños Privados. Madrid: Ediciones de la Torre, 1995.

APARICI, R. (coord.). La Revolución de los medios audiovisuales. Madrid: Ediciones De la Torre, 1993.

AREA, M. Los medios y las tecnologías en la educación. Madrid: Pirámide, 2004. 
BOLETÍN DEL CENTRO DE INVESTIGACIONES SOCIOLÓGICAS. "La televisión y los niños". Boletín $n^{\circ} 26$ [en línea]. Octubre-diciembre 2000, Estudio n 2.391. [Consulta: 21 de julio de 2006]. Disponible en http://www.cis.es/boletin/26/tele.htm

BUCKINGHAN, D. Educación en medios. Alfabetización, aprendizaje y cultura contemporánea. Barcelona: Paidós, 2005.

CABERO ALMENARA, J. (Coord.). Nuevas Tecnologías Aplicadas a la Educación. Madrid: Mc Graw Hill, 2007.

- (ed.) (2000) Nuevas tecnologías aplicadas a la educación. Barcelona: Síntesis.

CEBRIÁN HERREROS, M. Información multimedia. Madrid: Pearson, 2005.

- (2004) La información en televisión: obsesión mercantil y política. Barcelona: Gedisa.

- (2003) Análisis de la información audiovisual en las aulas. Madrid: Editorial Universitas.

- (1995) Información audiovisual: Concepto, Técnica, Expresión y Aplicaciones. Madrid: Síntesis.

GABELAS BARROSO, J. A.; MARTA LAZO, C. Consumo y mediaciones de familias y pantallas. Nuevos modelos y propuestas de convivencia. Zaragoza: Gobierno de Aragón, 2008.

GARCÍA MATILLA, A. Una televisión para la educación. La utopía posible. BarceIona: Gedisa, 2003.

GUTIÉRREZ MARTíN, A. Alfabetización digital. Algo más que ratones y teclas. Barcelona: Gedisa, 2003.

- (1997) Educación Multimedia y Nuevas Tecnologías. Madrid: Ediciones de la Torre.

MARTA LAZO, C.; GABELAS BARROSO, J. A. "La educación para el consumo de pantallas, como praxis holística". Revista Latina de Comunicación Social [en línea], $\mathrm{n}^{\circ} 62$ (2007). [Consulta: 15 de diciembre de 2007]. Disponible en http://www.ull.es/publicaciones/latina/200720_Carmen_Marta_Lazo.htm

MARTA LAZO, C. La televisión en la mirada de los niños. Madrid: Fragua, 2005.

MARTA LAZO, C.; DE ÁNGEL GARCÍA, N. "Análisis empírico sobre la decodificación del mensaje televisivo, según el proceso evolutivo en niños de clase media de 4 a 7 años", en AA. VV., Actas del I Congreso Internacional de Formación y Medios, Segovia, 7-10 julio 1997. Al cuidado de Alfonso Gutiérrez. Segovia: Escuela de Magisterio de Segovia, Universidad de Valladolid, 1997. pp: 327-333.

MINISTERIO DE EDUCACIÓN Y CIENCIA, Temas transversales y desarrollo curricular. Madrid: Ministerio de Educación y Ciencia, 1993. 
MORSY, Z. La Educación en Materia de Comunicación. París: UNESCO, 1984.

PÉREZ TORNERO, J. M. "El futuro de la sociedad digital y los nuevos valores de la educación en medios". Comunicar. Revista Científica Iberoamericana de Comunicación y Educación, (Noviembre 2005), n² 25, pp. 247-258.

- (Coord.) (2000). Comunicación y educación en la sociedad de la información. Barcelona: Paidós.

- (1994) El desafío educativo de la televisión. Para comprender y usar el medio. Barcelona: Paidós.

TELLO, J.; MONESCILLO, M. "La televisión como recurso curricular y medio de conocimiento". Comunicar. Revista Científica Iberoamericana de Comunicación y Educación, (Noviembre 2005), n² 25, pp. 231-236.

YOUNIS, J. A. El aula fuera del aula. La educación invisible de la cultura audiovisual. Las Palmas de Gran Canaria: Librería Nogal Ediciones, 1993.

\section{Breve semblanza de la autora}

Carmen Marta Lazo es profesora de la Facultad de Comunicación de la Universidad San Jorge de Zaragoza (España). Doctora en Ciencias de la Información por la Universidad Complutense de Madrid. Master en Televisión Educativa por la UCM y Master en Radio por RNE y UCM. Especialista en Nuevas Tecnologías y Educación por la UNED. Autora del libro La Televisión en la mirada de los niños de Fragua Comunicación, coautora de Consumos y mediaciones de familias y pantallas y de numerosos artículos en revistas científicas. Ha participado en varios proyectos de investigación centrados en el análisis de la audiencia infantil y adolescente de televisión y nuevas pantallas, financiados por el MEC, por la Universidad Complutense, por el Gobierno de Aragón y por la Fundación para la Investigación de la UFV, entre otros organismos.

(Recibido el 5-02-08, aceptado el 20-05-08) 